\title{
Novel data analysis approach for Raman Scattering-based Distributed Temperature Sensing (DTS) systems
}

\author{
by I. S. M. Shatarah*, B. Więcek* \\ * Lodz University of Technology, Institute of Electronics, 211/215 Wólczańska St. 90-924 Łódź, Poland, \\ iyad.shatarah@dokt.p.lodz.pl
}

\begin{abstract}
Distributed Temperature Sensing (DTS) systems based on optical fibers are well known for their ability to provide crucial information relative to temperature changes along the optical fiber, with a respectful temperature and spatial resolutions. Commercial DTS systems are commonly based on the Optical Time Domain Reflectometry (OTDR) and the Optical Frequency Domain Reflectometry (OFDR). This paper presents a novel analyzing method for DTS systems, based on the analysis of the backscattered light in the transition state along the optical fiber.
\end{abstract}

\section{Introduction}

The accuracy, practicality and quickness of the Distributed Temperature Sensing (DTS) systems have evaluated rapidly in the last decade, which led to higher popularity of these systems in several applications [1]. The production technology of the fiber optics has also evolved, resulting in enormous improvement in fibers' immunity and reliability in harsh environments [2]. The majority of the commercial DTS systems is based on the Optical Time Domain Reflectometry (OTDR) rather than the Optical Frequency Domain Reflectometry (OFDR). This is resulting from the simplicity of OTDR systems and relatively lower prices.

The main principle of the OTDR method is the measurement of attenuation of the backscattered light. OTDR based systems use short, powerful laser beams, which are launched into the optical fiber. The backscattered light is then detected, amplified and averaged, and the temperature profile along the optical fiber is then determined [3]. Meanwhile, OFDR based systems rely on the use of frequency modulated laser beams, and the backscattered light is detected as a function of the modulation frequency. Then, the application of IFFT is necessary in order to transform the obtained data to the spatial domain [4].

Both OTDR and OFDR systems are capable of detecting several hotspots in different locations along the optical fiber, with a respectful spatial and temperature resolutions. This paper presents a novel analyzing method for DTS systems. Initial experiments results indicate a high potential of this method. The simulation model has also provided the expected behavior of the DTS system in different conditions.

\section{A novel method of data analysis for DTS}

A series of experiments for a DTS system in a single-ended configuration, based on the OFDR analyzing method, was done using the set-up shown in figure 1. The presence of the optical circulator allowed driving the frequency modulated laser diode's beam into the optical fiber (from channel 1 into channel 2), and the backscattered light was led from the optical fiber to the wavelength division multiplexing (WDM) filter (from channel 2 into channel 3). The WDM filter is based on the passband filters, which are chosen in correspondence to the laser diode's wavelength, in order to separate the backscattered Rayleigh, Raman Stokes and Raman Anti-Stokes radiations [5]. Then, the avalanche photodiodes (APD) detected the backscattered radiations and their waveforms were observed on an oscilloscope. During the execution of measurements this DTS system, the backscattered Anti-Stokes radiation from the optical fiber, placed in temperature $T_{1}$, had the form presented in figure 2 . The results of these experiments showed clearly that the rise time of the backscattered Anti-Stokes radiation was:

$$
t=\frac{2 L}{c}
$$

where $L$ denotes the optical fiber length, $c$ denotes the light speed in vacuum. When light reached the end of the optical fiber, it had a high, constant value, resulting from the reflection of light at the end of the fiber. The result obtained during these measurements was the motive behind the idea of the novel analyzing method. 


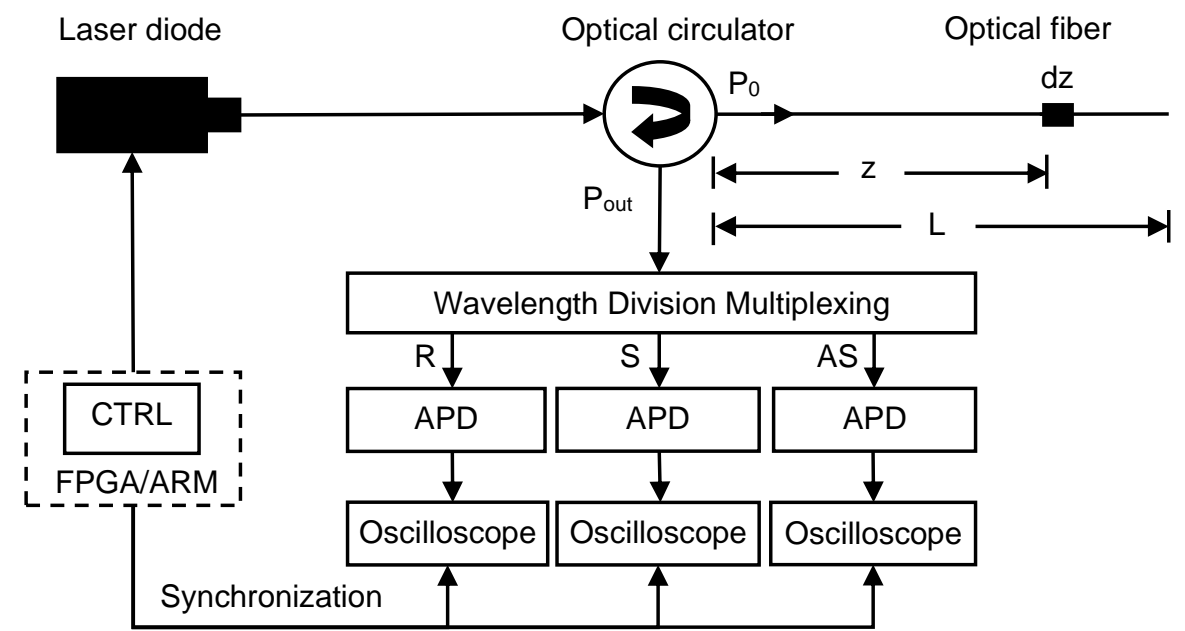

Fig. 1. DTS system set-up in single-ended configuration

The main point was examining the temperature effect on the rise time of the backscattered Anti-Stokes signal. The author assumed that temperature $T_{2}$ is higher than the temperature $T_{1}$, thus logically the backscattered Anti-Stokes signals for temperatures $T_{1}$ and $T_{2}$ should look as shown in figure 2. This results from the high dependency of Anti-Stokes backscattered radiation on temperature [1]. The laser diode was driven by a control unit, which allowed the setting of frequency. Knowing the length of the fiber and the speed of light in the fiber, the time, which light needed to reach the end of the fiber, was calculated. The main idea of the novel analyzing method is to consider the scattered light observed at the photodiode in the time that the light needs to reach the end of the fiber. Based on these facts, the mathematical simulation model was prepared.

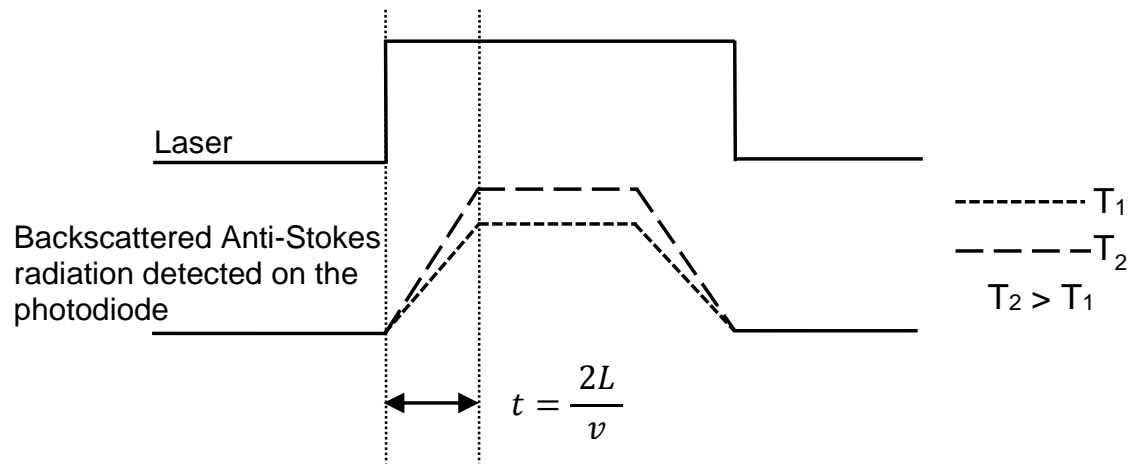

Fig. 2. The backscattered Anti-Stokes radiation waveform as observed on the oscilloscope at temperature $T_{1}$ and the novel method assumption for the backscattered Anti-Stokes waveform at temperature $T_{2}$

Assuming that the power of the launched laser beam is $P_{0}$, the power of the backscattered light should have the value presented in equation 2 :

$$
P(t, T)=P_{0} e^{-\alpha_{0} z} 1\left(t-z \frac{n_{0}}{c}\right)
$$

where $\alpha_{0}$ denotes the power attenuation of the laser wavelength corresponding the type of the optical fiber in the system set-up, and $n_{0}$ is the refractive index of the optical fiber for the laser's wavelength. In order to find the power of the Stokes or Anti-Stokes backscattered radiation at the section $\mathrm{dz}$ of the optical fiber length, equation 2 can be written in the form of equation 3 :

$$
\begin{gathered}
d P_{\text {out }, x}(t, T)=P_{0} \Gamma_{x} \rho_{x}(T) e^{-\alpha z} 1\left(t-z \frac{n}{c}\right) d z \\
\alpha=\alpha_{0}+\alpha_{x} \\
n=n_{0}+n_{x}
\end{gathered}
$$


where $x$ denotes Stokes (S) or Anti-Stokes (AS) backscattered radiation, $\Gamma$ denotes the capture coefficient, $\rho(T)$ denotes the Bose-Einstein statistical probability distribution of energy levels of the molecules [6]. Finally, the Laplace transform is applied in order to obtain the equations for the power of the backscattered Raman Stokes and Raman Anti-Stokes:

$$
\begin{gathered}
P_{\text {out }, x}(t, T)=P_{0} \Gamma_{x} \rho_{x}(T) \int_{0}^{L} e^{-\alpha z} 1\left(t-z \frac{n}{c}\right) d z \\
F(s)=\int_{0}^{\infty} f(t) e^{-s t} d t \\
P_{\text {out }, x}(s, T)=P_{0} \Gamma_{x} \rho_{x}(T) \int_{0}^{L}\left\{e^{-\alpha z} \int_{0}^{\infty} 1\left(t-z \frac{n}{c}\right) e^{-s t} d t\right\} d z \\
P_{\text {out }, x}(s, T)=\frac{P_{0} \Gamma_{x} \rho_{x}(T)}{s} \int_{0}^{L} e^{-\left(\alpha+\beta_{x} s\right) z} d z \\
\beta_{x}=\frac{n}{c} \\
P_{\text {out }, x}(s, T)=\frac{P_{0} \Gamma_{x} \rho_{x}(T)}{s\left(\alpha+\beta_{x} s\right)}\left[1-e^{-\left(\alpha+\beta_{x} s\right) L}\right] \\
k_{x}(s, T)=\frac{\Gamma_{x} \rho_{x}(T)}{\alpha+\beta_{x} s}\left[1-e^{-\left(\alpha+\beta_{x} s\right) L}\right]
\end{gathered}
$$

The novel analyzing method provided in this model could be considered as a combination of the both wellknown analyzing methods: OTDR and OFDR. The proposed method uses frequency modulated laser beams, and applies the calculation algorithm in the time domain, measuring the backscattered signals during the time they need to travel from the scattering point (the hotspot).

\subsection{Model parameters}

The model presented in this paper was prepared for a $1000 \mathrm{~m}$ single-mode optical fiber. The laser diode wavelength was chosen to be $1550 \mathrm{~nm}$, and the modulation frequency range is $0-100 \mathrm{MHz}$. However, the model is applicable to other types of optical fibers and several other laser's wavelengths. The set-up configuration and parameters depend on the application, the desired spatial resolution and spatial range of the DTS system. Therefore, the modelling of the DTS system and the comparison of the results is essential before the actual build of the DTS system.

Table 1. DTS system parameters used in this model

\begin{tabular}{|l|l|c|}
\hline \multirow{2}{*}{ Laser diode } & Power & $20 \mathrm{~mW}$ \\
\cline { 2 - 3 } & Wavelength & $1550 \mathrm{~nm}$ \\
\hline \multirow{3}{*}{ Optical fiber } & Type & SMF 28-e \\
\cline { 2 - 3 } & Length & $1000 \mathrm{~m}$ \\
\hline \multirow{4}{*}{ Raman Stokes } & Wavelength & $1660 \mathrm{~nm}$ \\
\cline { 2 - 3 } & Power attenuation & $0.22 \mathrm{~dB} / \mathrm{km}^{-1}$ \\
\cline { 2 - 3 } & Capture coefficient & $3.04 \mathrm{~m}^{-1}$ \\
\hline \multirow{3}{*}{ Ramti-Stokes } & Wavelength & $1450 \mathrm{~nm}$ \\
\cline { 2 - 3 } & Power attenuation & $0.25 \mathrm{~dB} / \mathrm{km}^{-1}$ \\
\cline { 2 - 3 } & Capture coefficient $\left(\mathrm{m}^{-1}\right)$ & $4 \mathrm{~m}{ }^{-1}$ \\
\hline
\end{tabular}

\subsection{Model results}

Figures $3-10$ present some cases of possible measurments for a DTS system. The results presented in the figures are for the Anti-Stokes backscattered radiation, which is the most dependent type of ligth scattering on temperature []. Figure 3 presents the results for a DTS system, where the temperature along the whole optical fiber is increased. The power of the backscattered ligth is expected to be high due to the high scattering level, which is occaring because of the higher temperature along the whole length of the optical fiber. Figure 4 presents the difference in the power between the inaital temperature state and the higher temperature states. 


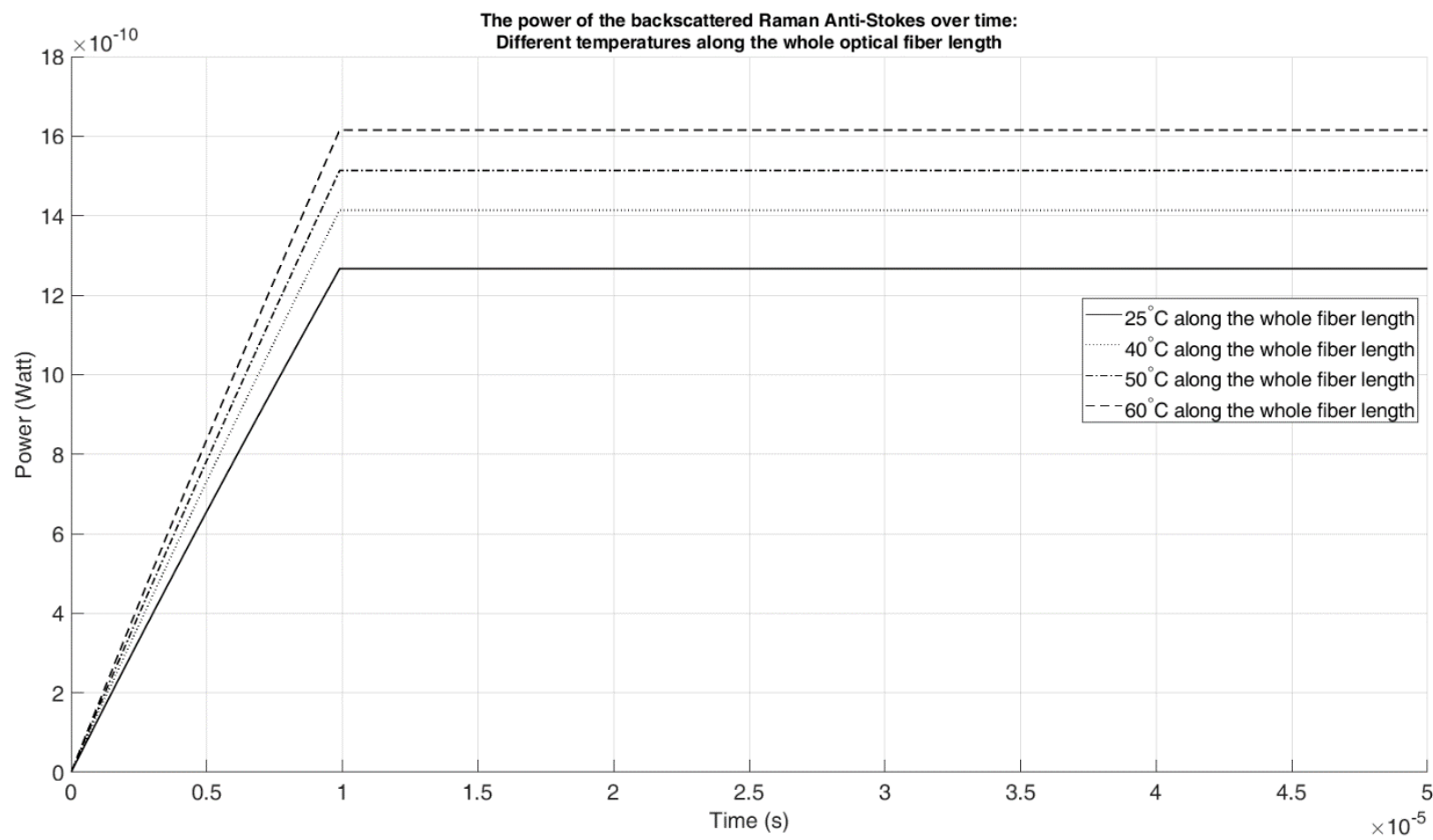

Fig. 3. The power of the backscattered Anti-Stokes radiation for different temperature profiles along the whole optical fiber

The power difference of the backscattered Raman Anti-Stokes over time:

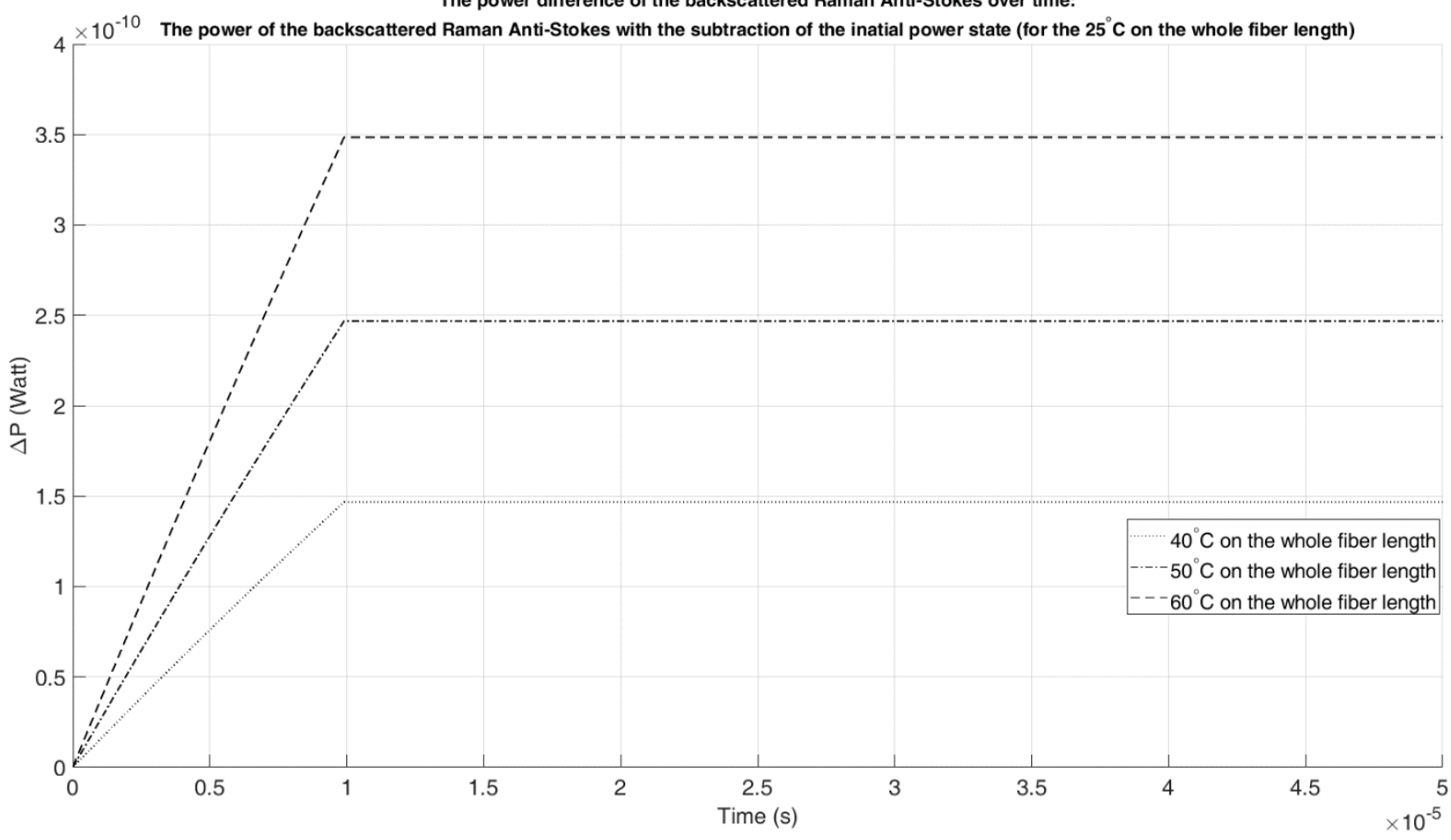

Fig. 4. The power difference between the the inaital temperature state and the higher temperature states for the case presented in figure 3 
Figures 5 and 6 present the results for a DTS system, in which the temperature is increased at the last 10 meters of the optical fiber. The power of the backscattered Anti-Stokes signals is constant along the fiber, yet it rises at the last 10 meters depending on the temperature.

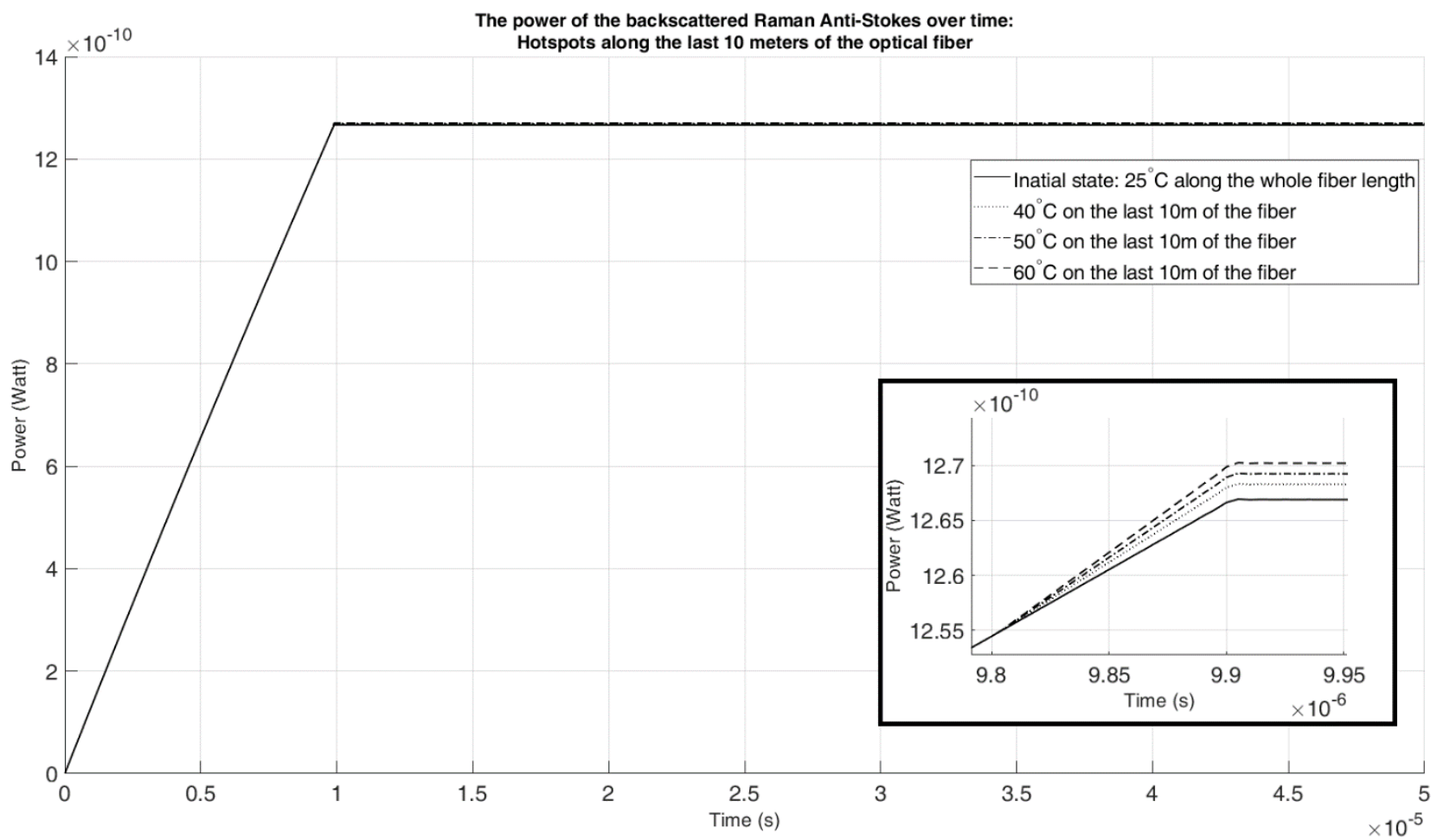

Fig. 5. The power of the backscattered Anti-Stokes radiation for different temperature profiles along the last 10 meters of the optical fiber

The power difference of the backscattered Raman Anti-Stokes over time:

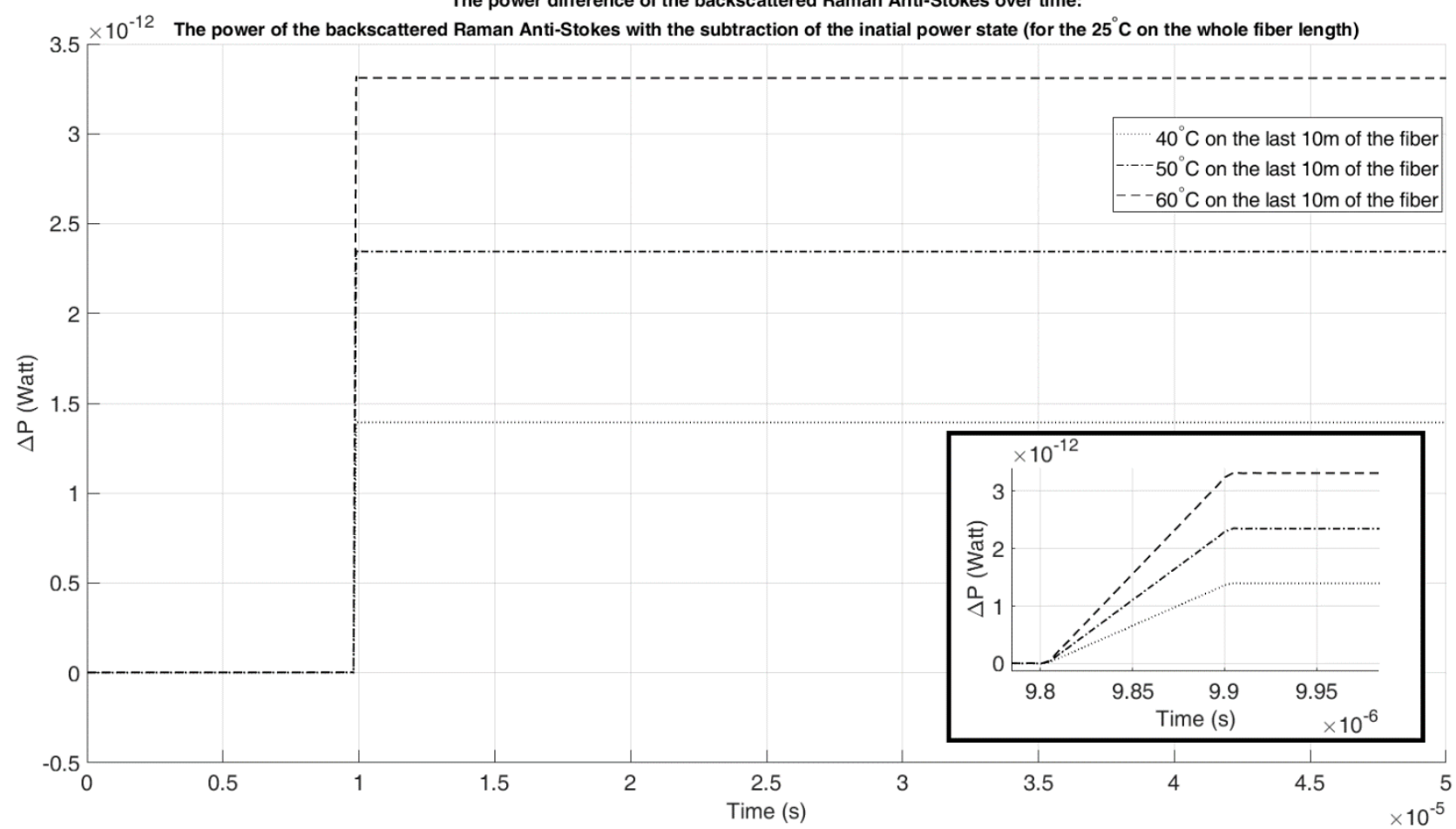

Fig. 6. The power difference between the the inaital temperature state and the higher temperature states for the case presented in figure 5 
Figures 7 and 8 present the case, where the temperature is increased along two different ranges in the middle of the optical fiber. The temperature increase is the same for both lengths. The longer the range incused to the high temperature, the higher the power of the Anti-Stokes backscattered radiation.

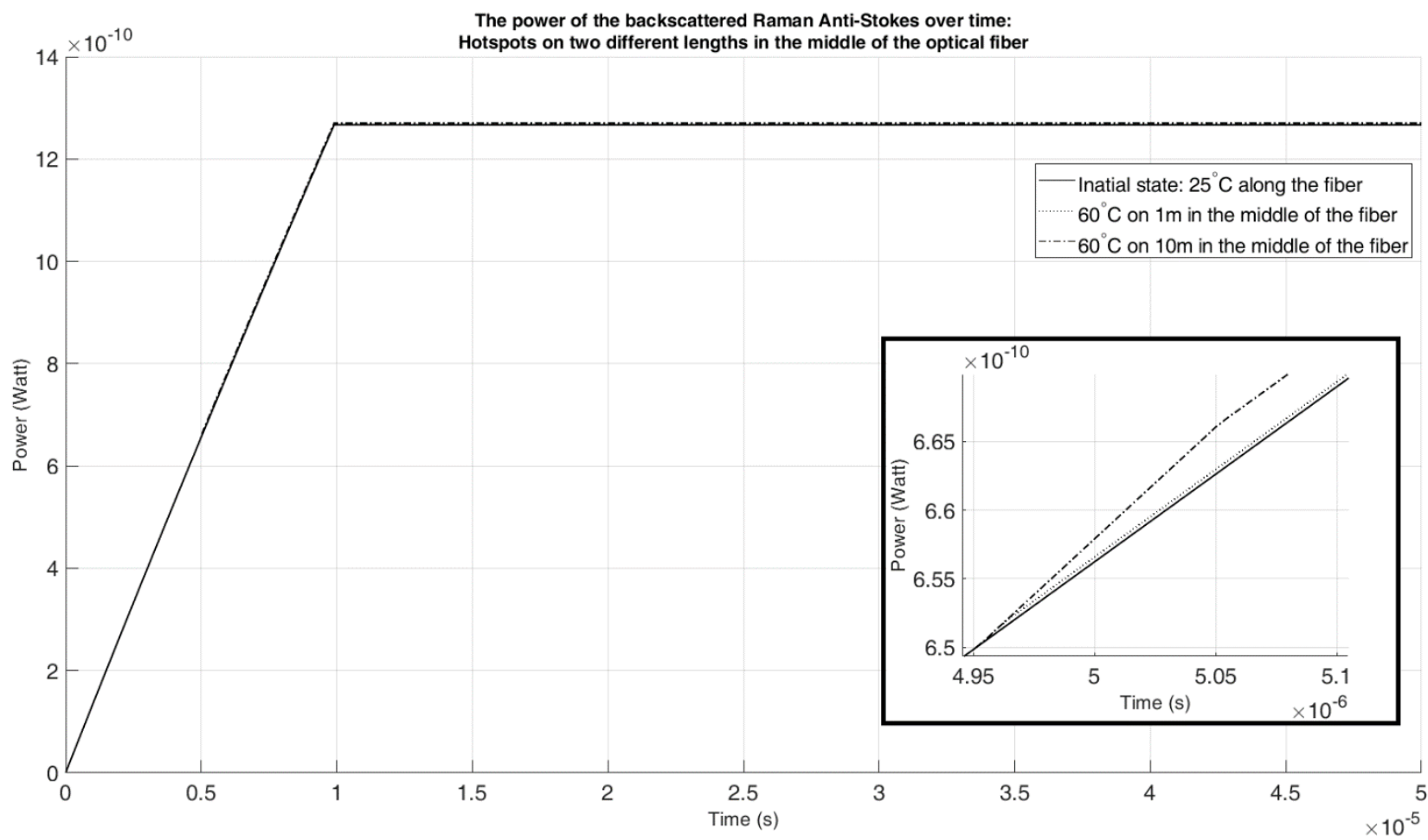

Fig. 7. The power of the backscattered Anti-Stokes radiation for different temperature profiles along 1 meter and 10 meters in the middle of the optical fiber

The power difference of the backscattered Raman Anti-Stokes over time:

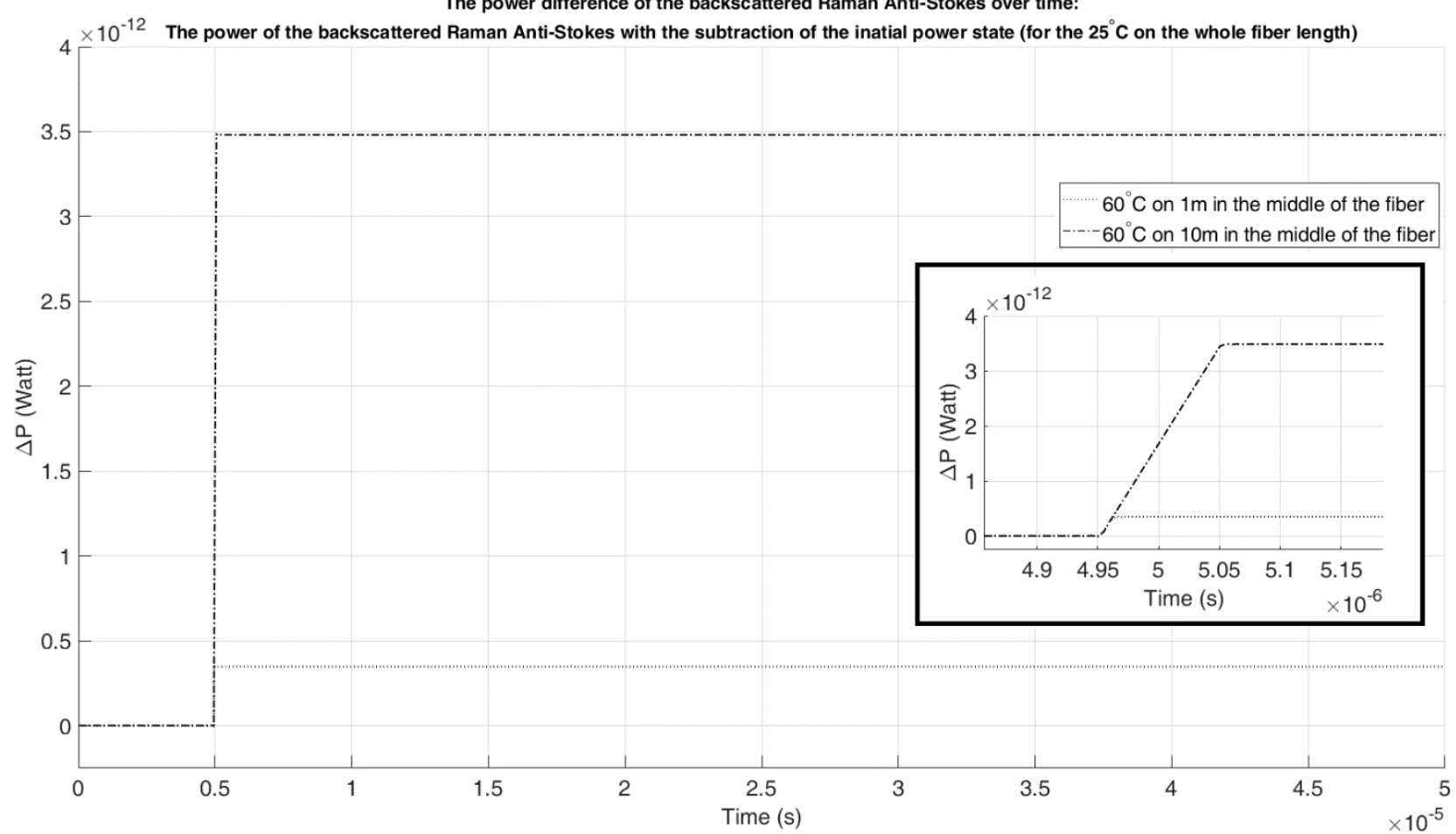

Fig. 8. The power difference between the the inaital temperature state and the higher temperature states for the case presented in figure 7 
Figures 9 and 10 present a combination of temperature increase on the middle and on the end of the optical fiber. The anti-Stokes backscattered signals power rises then stabilizes until the temperature is raised again at the end of the optical fiber.

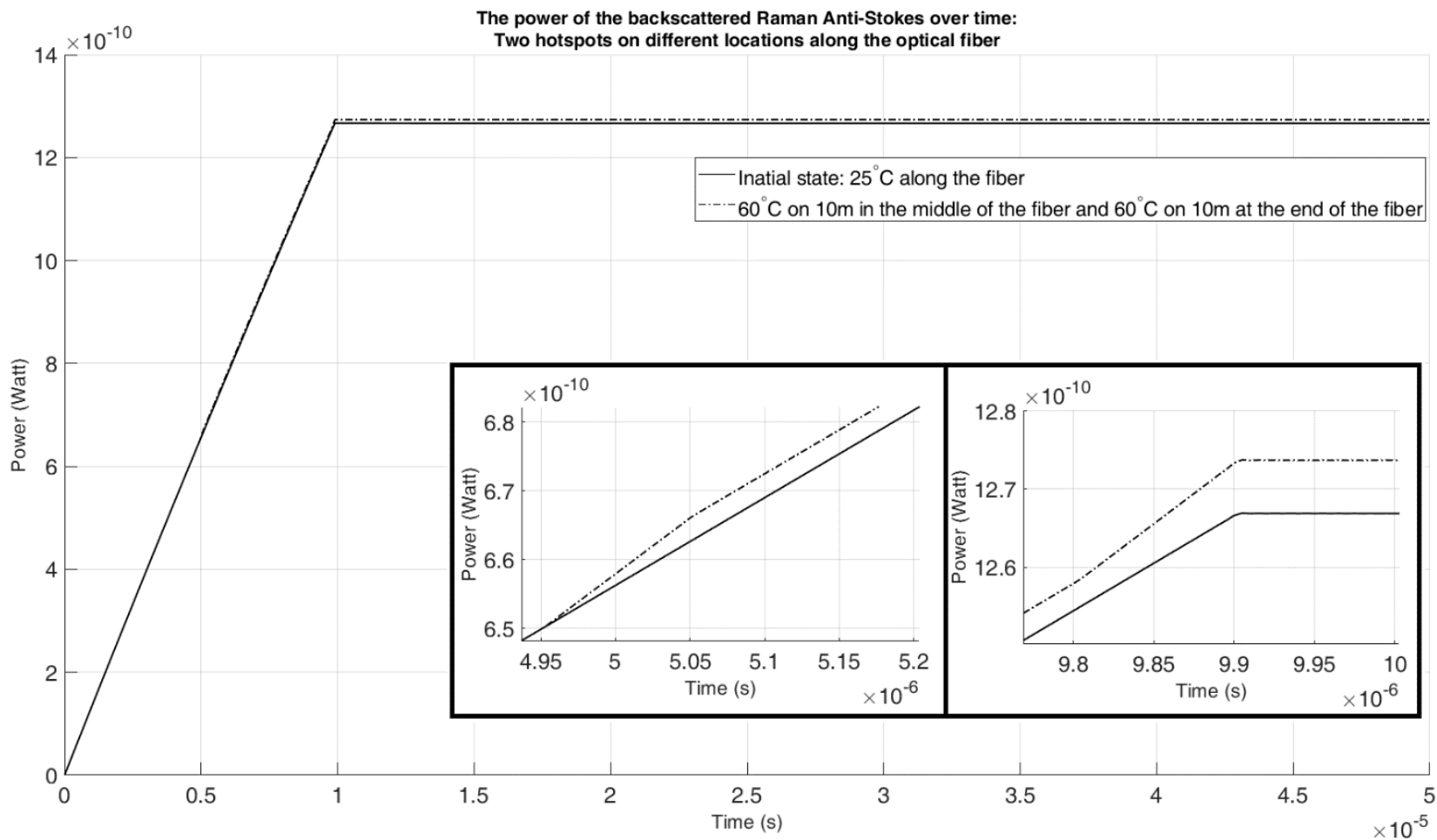

Fig. 9. The power of the backscattered Anti-Stokes radiation for a combination of temperature increase in the middle and at the end of the optical fiber

The power difference of the backscattered Raman Anti-Stokes over time:

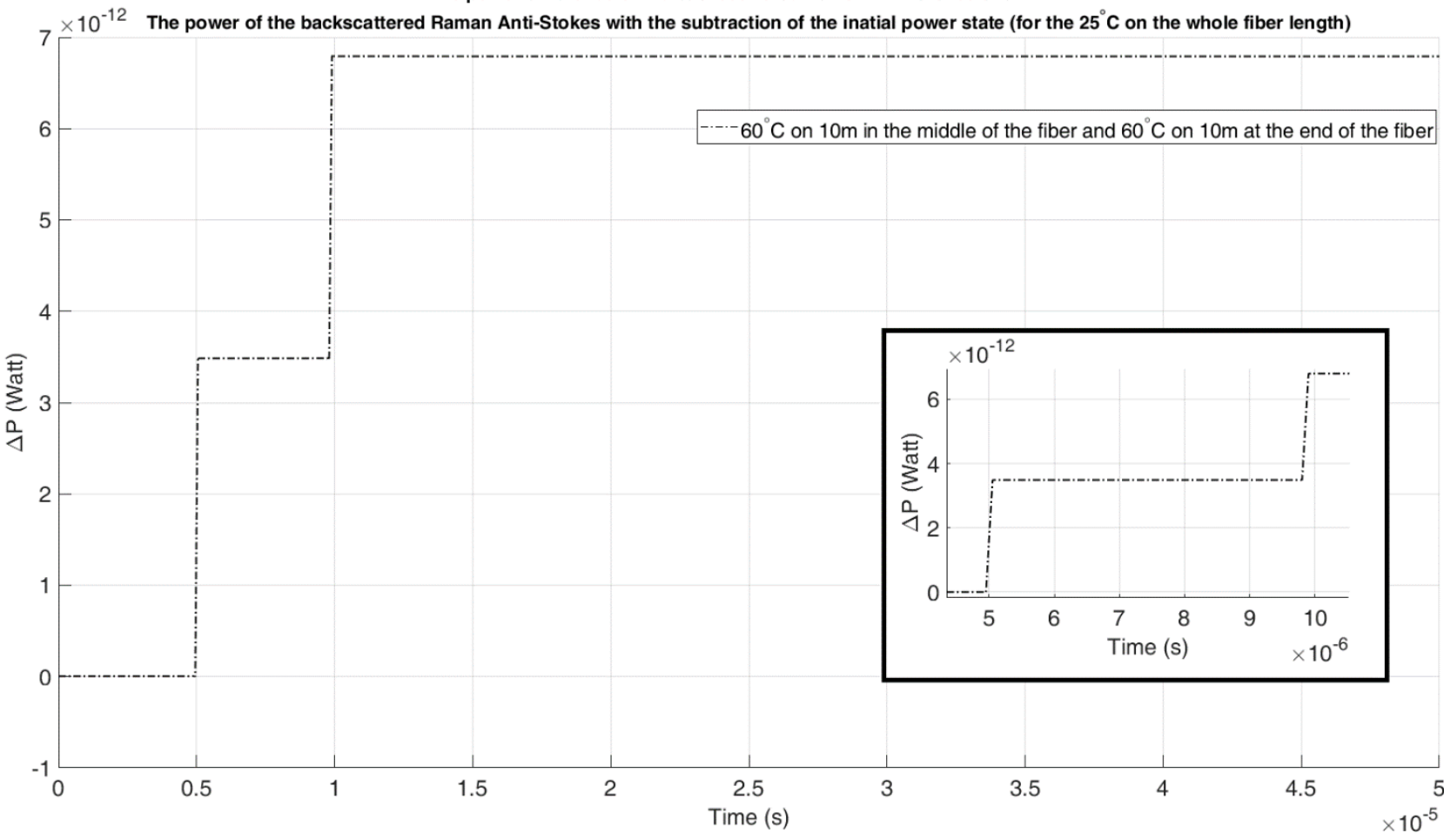

Fig. 10. The power difference between the the inaital temperature state and the higher temperature states for the case presented in figure 9 


\section{Conclusion}

This paper presented a novel method of data analysis for DTS systems. The mathematical model estimates the expected power of Stokes and Anti-Stokes backscattered radiations for various cases of possible measurements. The results shown in this paper were pertained to specific laser power and wavelength, and for a single-mode optical fiber. The model can easily be applied for different types of lasers and optical fibers intended to be used in the DTS system. Based on the results shown in figures 3-10, one can estimate the power of the backscattered radiation and the capabilities of the DTS system to detect the temperature change along the fiber. The power change ratio can also be estimated. Moreover, this model helps providing the crucial information regarding the best components to be integrated in the DTS system depending on the application and the intended spatial and temperature resolutions.

\section{REFERENCES}

[1] Shatarah I. S. M., Więcek B., The application of NIR spectrometer for average temperature measurement in optical fibers based on spontaneous Raman scattering for DTS applications. MIXDES 2020 Conference Proceedings, Poland, 2020.

[2] Seitz W. R., Chemical Sensors Based on Fiber Optics. Analytical Chemistry, vol. 56, no. 1, pp. 16 - 22, 1984.

[3] Hartog A. H., An Introduction to Distributed Optical Fiber Sensors. CRC Press, Boca Raton, ISBN 9781138082694, 2017.

[4] Farahani M. A. and Gogolla T., Spontaneous Raman Scattering in Optical Fibers with Modulated Probe Light for Distributed Temperature Raman Remote Sensing. Journal of Lightwave Technology, vol. 17, no. 8, pp.13791391, 1999.

[5] Senior J. M., Optical fiber communications: Principles and practice. Pearson Education Limited, Harlow, England, 2009.

[6] I.S.M Shatarah, R. Olbrycht and B. Więcek, Modeling of Spontaneous Raman Scattering in silica light guides for Distributed Temperature Sensing. 14th Quantitative Infrared Thermography Conference (QIRT), Berlin 2018, pp. 209-220. 